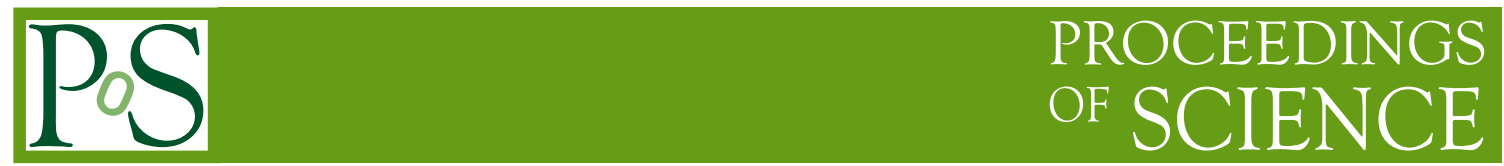

\title{
Data Readiness of the LHCb Vertex Locator (VELO)
}

\author{
Karol Hennessy*i \\ University of Liverpool \\ E-mail: karol.hennessyecern.ch
}

The Vertex Locator (VELO) is the primary vertexing and tracking detector for LHCb. It is a silicon microstrip detector and surrounds the primary interaction point at LHCb. A study of the performance of the detector using particles from LHC injection tests is detailed here. The VELO was found to have good spatial and time alignment. The signal-to-noise and resolution performance were close to expectation, and overall the detector was determined to be ready for physics-quality data taking.

VERTEX 2009 (18th workshop) - VERTEX 2009

September 13 - 182009

Veluwe, the Netherlands

* Speaker.

On behalf of the LHCb VELO group 


\section{Introduction}

LHCb is a flavour physics detector, designed to detect decays of b- and c-hadrons for the study of CP violation and rare decays[1]. CP violation in the Standard Model does not account for the abundance of matter over anti-matter in the universe. LHCb is the only dedicated hadron collider b-physics experiment currently in operation.

The Vertex Locator (VELO) is the primary vertexing and tracking detector for $\mathrm{LHCb}[2]$. The VELO surrounds the interaction point in $\mathrm{LHCb}$, and has to provide precise measurements of track coordinates close to the interaction region.

\section{The LHCb Vertex Locator}

The VELO consists of 42 modules placed along the beam direction, with the full length of the detector being approximately $1 \mathrm{~m}$. The mean interaction point is approximately $18 \mathrm{~cm}$ from the first VELO module - meaning more modules sit in the forward region. Fig. 1 shows a cut-away view of the VELO. The modules can be seen in the centre of the tank. An exploded view of a VELO module can be seen in fig. $2 \mathrm{a}$, and in fig. $2 \mathrm{~b}$ is a photograph of a fully mounted module.

The VELO modules consist of the two silicon sensors, $\mathrm{R}$ and $\phi$, and 32 Beetle chips bonded to a TPG (Thermal Pyrolytic Graphic) hybrid. This, in turn, is mounted onto a rigid carbon fibre pedestal. Kapton Cables are used to connect the modules to the Repeater boards. A bi-phase $\mathrm{CO}_{2}$ cooling block is attached to one side to cool the module to approximately $-10^{\circ} \mathrm{C}$ when fully powered. The modules are positioned to within $8 \mathrm{~mm}$ of the beam and are retractable to protect the sensors, as the LHC beam is less stable during injection.

Each sensor has 2048 strips. The R sensors have circular arcs of strips, and are split into 4 quadrants of 512 strips each. The strip pitch varies from $40 \mu \mathrm{m}$ in the innermost region to $101.6 \mu \mathrm{m}$

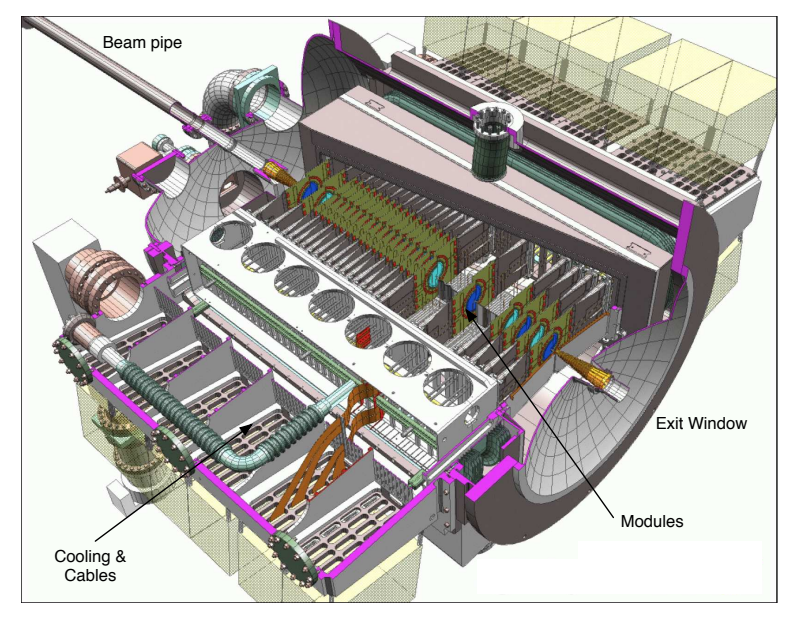

Figure 1: VELO vacuum tank housing 42 VELO modules. A $300 \mu \mathrm{m}$ aluminium foil separates the VELO vacuum from the primary LHC vacuum. Each VELO half is moved out a distance of $29 \mathrm{~mm}$ from the beam line during injection. 


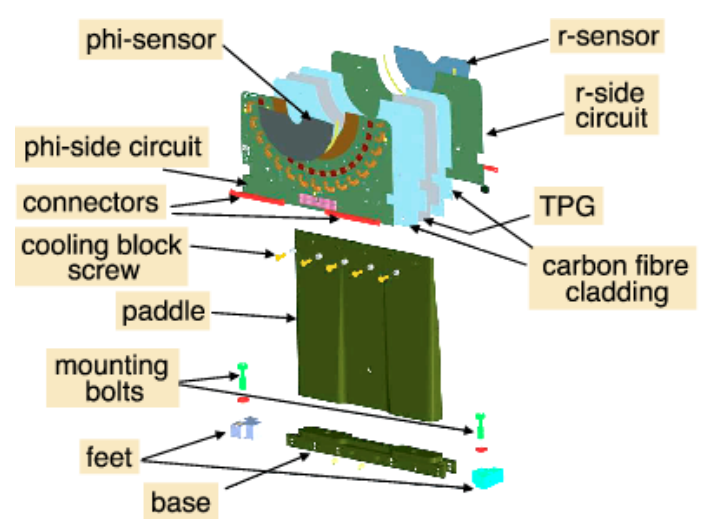

(a)

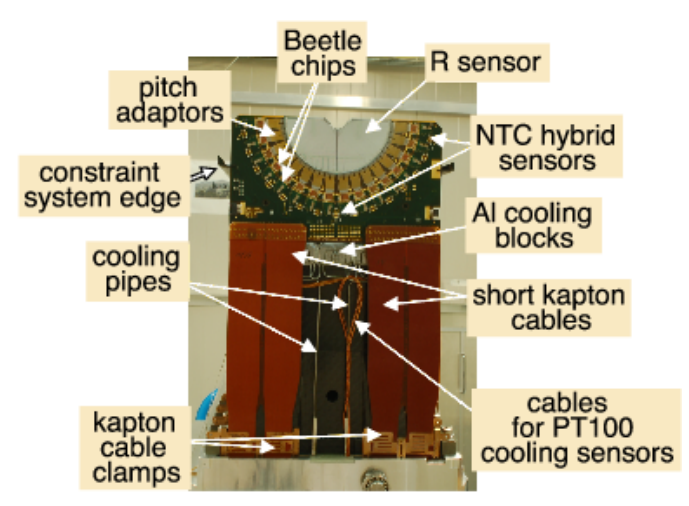

(b)

Figure 2: (a) Exploded view of a double-sided VELO module with R and $\phi$ sensors shown. (b) Photograph of a mounted VELO module.

in the outermost region. The $\phi$ sensors have two kinds of strips - inner strips with a pitch of $35 \mu \mathrm{m}$ and outer strips with a pitch of $101 \mu \mathrm{m}$.

\subsection{VELO Electronics Readout Chain}

The Beetle front-end chips [3] are responsible for transmitting the charge collected on the silicon strips to the readout electronics. Each chip has four data output lines (known as links), and each link reads out 32 strips and appends 4 header channels to the analogue signal sent to the readout chain.

Next in the chain are the repeater boards, which shape and amplify the analogue data signals from the Beetle chips and send them to the Tell1 boards over $60 \mathrm{~m}$ of differential analogue copper cables. The Tell1 [5] is a multi-purpose readout board for LHCb. For the VELO, it consists of four ARx cards for analogue to digital conversion. The digitised signals are processed by FPGAs on the board which zero-suppress the data. Zero-supression involves a series of algorithms designed to extract particle hits in the data from background noise. Also on board the Tell1 are a Credit-Card PC which communicates with the Experimental Control System, and a Gigabit Ethernet card for sending the zero-suppressed data to the High Level Trigger farm.

\subsection{Radiation Hardness}

In LHCb, the high levels of radiation are a concern. When fully closed, the VELO modules are just $8 \mathrm{~mm}$ from the beam line, making the environment particularly hostile. For the VELO, $\mathrm{n}^{+}$strips in n-bulk silicon was chosen, as the depleted layer is on the same side as the strips. This means that the sensors are radiation hard - they can retain $90 \%$ of their functionality after 3 years of LHCb running. Fig. 3a is a plot from a half-irradiated sensor showing how collected charge varies with fluence. Fig. $3 b$ shows the depletion voltage of a sensor as a function of radius after one year of nominal LHC running. A replacement VELO is currently being built, and will be installed during one of the LHC shutdown periods. 


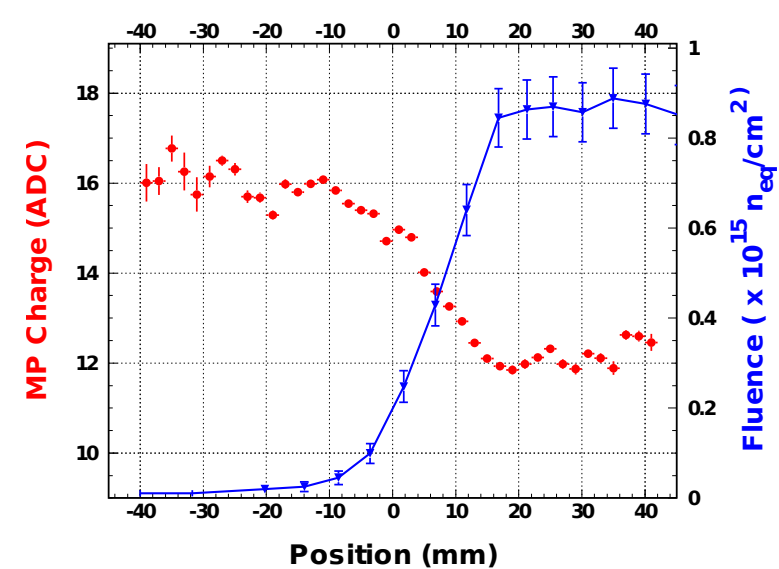

(a)

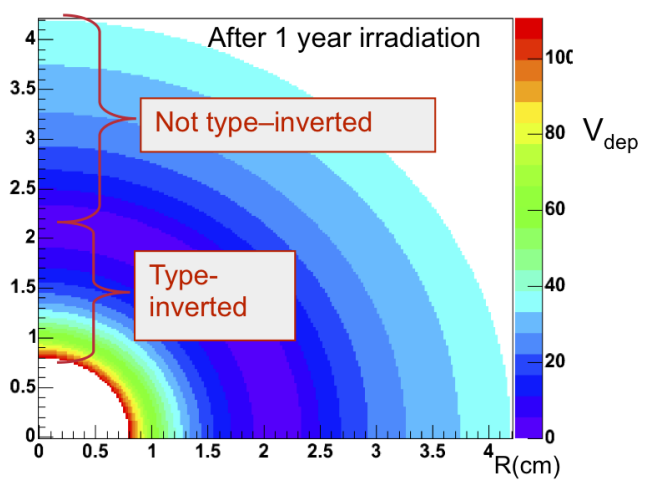

(b)

Figure 3: Radiation Hardness of the VELO sensors. (a)Plot of charge collected a sensor with differing degrees of irradiation. The right hand side received the largest dose and the charge collection efficiency shows a clear decrease on this side of the sensor. (Credit - JC Wang, Syracuse) (b)Plot of depletion voltage vs. radius. After one year at $2 \mathrm{fb}^{-1}$, the inner zone receives the highest dose and becomes type-inverted, while the outer region remains non-inverted.

\section{Detector Performance}

\subsection{TED}

TED stands for "Transfer line External beam Dump". This is a beam dump approximately $300 \mathrm{~m}$ downstream of LHCb, just off the injection line from the SPS to the LHC. The SPS accelerates protons to $450 \mathrm{GeV}$, and the ensuing shower from these protons hitting the TED project in the direction of LHCb. These showering particles were used in testing and calibrating the detector.

Periods of data taking involving TED events took place four times - once in 2008 and three times in 2009. These proceedings describe TED events from August 2009. The object of this TED data taking period with respect to the VELO, was to use the showering particles to time and space align the detector, and calculate the signal-to-noise, efficiency, resolution of the sensors. The following sections detail the results of these analyses.

\subsection{Timing}

The VELO has two timings, or delays that are used to synchronise the VELO readout. There is the delay used to adjust to time-of-flight of the particles crossing the sensors and and the delay in the propogation of data through the read-out chain. We correct for the latter first, by adjusting the digitisation timing of the ADC cards on the Tell1s. The time-of-flight correction is corrected by adjusting the Beetle sampling time.

The digitisation timing is the time for the signals to travel from the Beetle chips to the Tell1s. In theory, setting the digitisation timing only has to be performed once for the lifetime of the experiment (as it is mostly based on cable length). However, in practice, components fail and have to be replaced, and new timings must be calculated for the new hardware. 


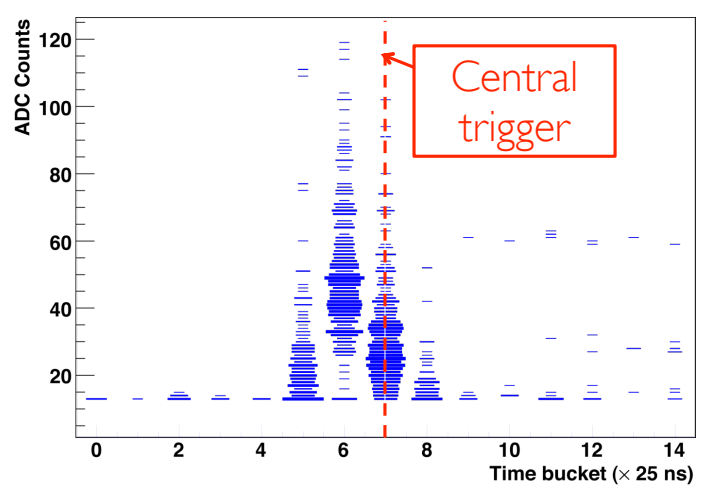

(a) Coarse Timing

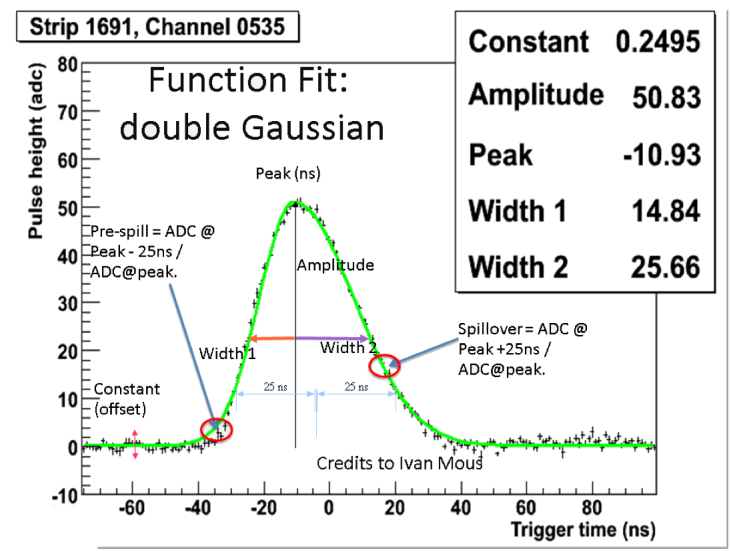

(b) Fine Timing

Figure 4: Determining the coarse and fine timings.

The digitisation timing is independent of the beam and can be calculated using calibration pulses sent from the beetle chips. The digitisation timing is essential both for data taking for physics and testing. This timing has been well tested and a procedure has been put in place to perform a timing scan and extract a new set of optimal digitisation timings.

The Beetle sampling time reflects the delay between a trigger being sent and the time the corresponding particles cross a VELO sensor. The Beetle sampling time must be adjusted for each sensor due to different time-of-flight for each. The VELO has a rather small active area, making timing using cosmic particles unfeasible. Therefore, the August TED period was a good opportunity to accurately test the Beetle sampling timing procedure.

Setting the Beetle sampling time is a two step process. The first step was to estimate the coarse delays, based on cable lengths and known delays in the system. At nominal running, the LHC collides bunches every $25 \mathrm{~ns}$. We can look in adjacent $25 \mathrm{~ns}$ time buckets to our "best guess" to determine our coarse timing. Fig. 4a shows a plot of ADC counts versus time bucket. Our "best guess" is shown in the centre. Based on the plot, one would estimate the timing to be somewhere between the central bin and the previous one. This estimate is used as a starting point for the fine timing scan. To determine the fine Beetle sampling time, we examine the Beetle pulse shape. This is performed in a similar manner to the coarse timing. By looking at the ADC counts in smaller time buckets, one can map out the Beetle pulse shape. Typically, four time steps are taken (4, 6.5, $12.5,19 \mathrm{~ns}$ ) and a function is fitted to determine the peak of the pulse. Fig. $4 \mathrm{~b}$, shows the Beetle pulse shape with $1 \mathrm{~ns}$ time buckets and the functional fit. As can be seen in the figure, the width of the pulse is greater than $25 \mathrm{~ns}$. To minimise the amount of signal spilling into the next (spillover) and previous events (pre-spill), we choose a timing $4 \mathrm{~ns}$ after the peak of the pulse.

\subsection{Signal and Noise Performance}

Using the particles from TED, the signal to noise was calculated. In ADC counts, the Most Probable Value (MPV) of a Landau from design is 35.4 for $\phi$ sensors and 34.0 for R sensors. The results obtained were 34.4 for $\phi$ sensors and 32.8 for R sensors, within $97 \%$ of expectation. The discrepancy can be explained by slight mis-timing, or gain mis-normalisations etc. These are, by no 


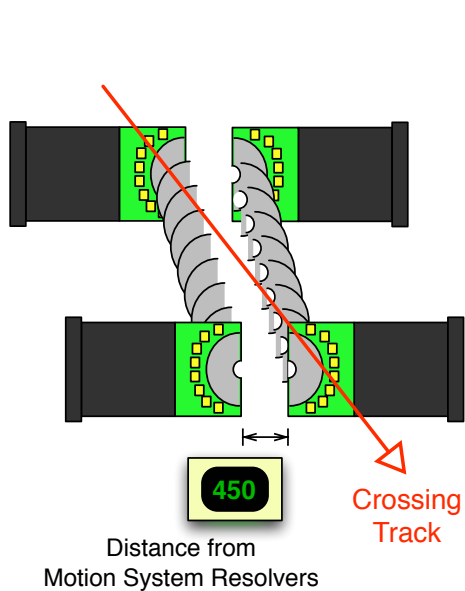

(a)

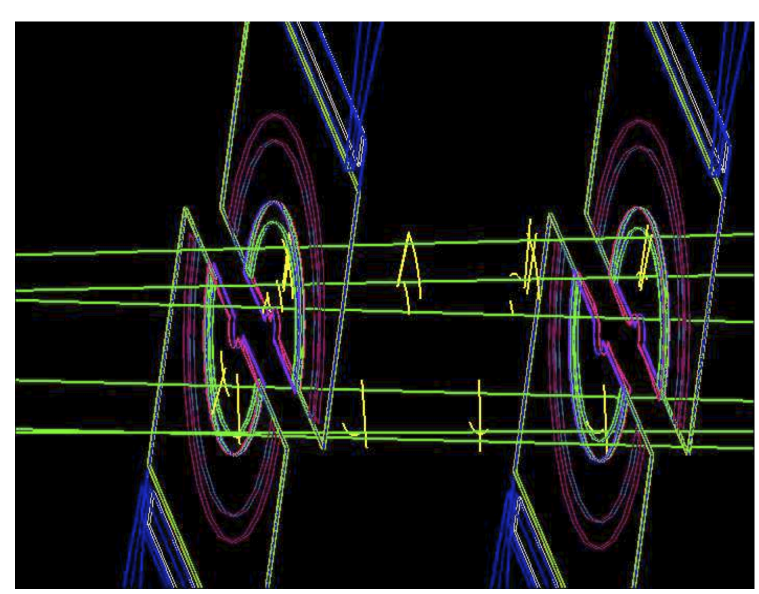

(b)

Figure 5: (a) Cartoon of a track crossing both VELO halves. Separation can be obtained from the motion system resolvers. (b) Visualisation of reconstructed tracks from a TED event. Four wire-frame models of the VELO modules are shown for clarity. Yellow arcs and lines are R and $\phi$ clusters respectively.

means, final numbers for the detector, and will be recalculated with data from LHC collisions. Prior to the TED data taking run, the gain of the ADC cards was normalised for VELO. The gain was calibrated using header information from the Beetle chips, such that a minimum ionising particle produces the same value in ADC counts throughout the detector.

The determined Signal-to-Noise values were slightly lower than expected. As yet, it is not clear if this is inherent in the system and removable.

\subsection{Spatial Alignment}

The results from the spatial alignment analysis using TED data are very encouraging [7]. Module Alignment constants were compared between TED data sets from 2008 and 2009. The constants agreed to $1.2 \mu \mathrm{m}$. The detector displacement from the values determined by metrology was less than $10 \mu \mathrm{m}$.

A test of the alignment procedure was performed using the VELO motion system. The VELO was moved from position (1) to a new position (2). These positions were reported via the motion system resolvers. In order to calculate the difference between the two VELO halves using the alignment framework, tracks crossing both VELO halves had to be used. 225 tracks were used to determine position (1) and 852 tracks were used to determine position (2). The expected value as measured using the motion system resolvers was $450 \mu \mathrm{m}$. The results indicated that the VELO had moved by $445 \pm 10 \mu \mathrm{m}$, in very good agreement with expectation.

\subsection{Resolution}

The resolution of two sample sensors ( $\mathrm{R}$ and $\phi$ ) calculated from TED events is given in fig. 6 . The resolution is determined from track residuals. The plots show approximately binary resolution. Binary resolution is the theoretical best resolution that could be achieved from a detector equivalent to the VELO, but with digital readout (where that resolution would be $\frac{\text { strippitch }}{\sqrt{12}}$. However, VELO 
Sensor Number 34

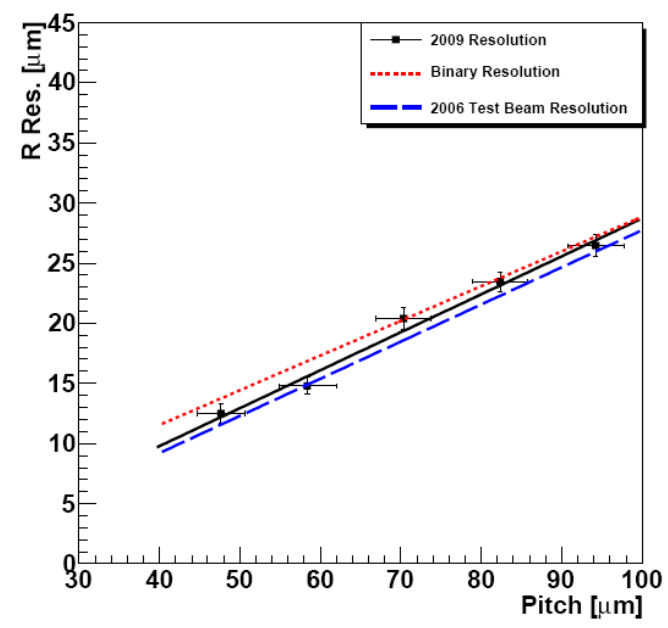

(a) R sensor (preliminary)
Sensor Number 77

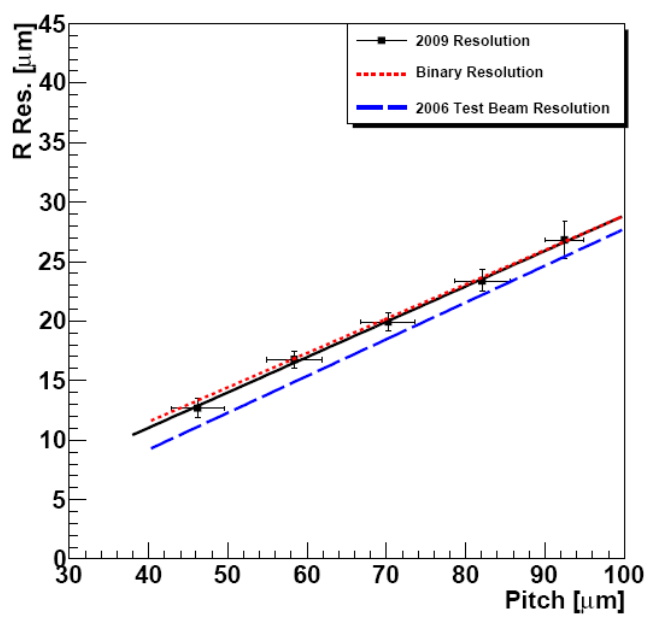

(b) $\phi$ sensor (preliminary)

Figure 6: Resolution as a function of strip pitch. Two example sensors are shown, an R and a $\phi$. The overall resolution is approximately equal to binary.

is an analogue detector, therefore, one would expect better than binary resolution. There are a couple of possible sources of poorer than expected resolution. Firstly, the momentum distribution of tracks from the TED is not well known. If the particles have low momenta, there may be multiple scattering in the detector, so the particles may not be perfectly straight, and poorer resolution is to be expected. Furthermore, the tracks used in the analysis of TED data are almost parallel to the beam line, hence there is less charge sharing, and the resolution becomes poorer.

\subsection{Monitoring}

The accident with the LHC in September 2008 afforded some time to improve the monitoring for the VELO. Much work has been done in this area over the past year.

An offline monitoring GUI has been developed for monitoring the following: (i) Noise, (ii) Pedestals, (iii) Occupancies, (iv) Bad Channels, (v) Cross-talk, (vi) Clusters, (vii) Gain, (viii) Tracks, (ix) IV Curves.

A complete description of these elements is beyond the scope of this document. Nonetheless, these tools make an invaluable method of monitoring the performance of the VELO. In terms of online monitoring, many of the plots used in the offline monitoring GUI are duplicated in the online monitoring interface (or "presenter" as it is known). The focus of the online monitoring is somewhat different however, as the safety of the detector is of primary importance. Changes in cluster occupancies and numbers of errors would indicate possible problems with the detector or beam-induced accidents. A separate panel is used to monitor voltages, currents, temperatures, vacuum pressures and beam position.

\subsection{Readout Tests}

High rate tests of the VELO and the LHCb detector as a whole have been performed and continue to be performed at regular intervals. These tests provide a measure of the overall system 
health. In order for these tests to be successful, not only do the readout electronics need to perform well at high data rates, but the detector itself must be performing within expected norms. Excess noise contributing to cluster occupancies can throttle the data rate. If the system is not properly synchronised or un-tuned configuration parameters have been uploaded, error banks will be produced by the Tell1s and again the data rate will suffer.

The design operating rate from the L0 Hardware Trigger is 1MHz [1]. With VELO and most of the other sub-detectors on the experiment included, a data rate of $981 \mathrm{kHz}$ was achieved.

\section{Difficulties Encountered}

This section describes the recurring problems that may have an impact on data taking as the experiment prepares for physics.

Low Voltage - The LV system has had many issues - including voltage trips, poor connectivity, and current read-back problems. Most of these issues have been resolved or a workaround has been put in place. In the last year, two LV modules out of 21 have been replaced/repaired and one bulk supply (equivalent to half of VELO). Repairs from manufacturer have been slow. Spares have been made available to cope with possible LV failures in the future.

High Voltage - The HV system has had problems of a different nature. These are mostly software related. Control signals to ramp the voltage up or down often need to be sent more than once. Error flags are sometimes sent by the hardware when the voltage is off. Hardware interlocks have been put in place in case of software failure.

Tell1 Readout Board - There are 84 Tell1s for the VELO. These are a multipurpose readout board for the experiment. Some have been known to fail after a power-cut. Some have failed in high-rate tests. Some of the Tell1s have had manufacturing problems, which have since been cured. Extensive testing of the ADC mezzanine cards is necessary before installation. Fully tested spares have been made available.

Cabling - VELO has probably the last large scale copper readout system. As such a large number of densely packed analogue data cables connect the VELO to the Tell1 boards. Care must be taken during replacement.

\section{Conclusions}

The VELO has shows a good signal-to-noise within a few percent of expectation. The spatial alignment shows very good agreement with the metrology. The operation of fine timing the detector to beam was successful. High rate tests show that the system as a whole can achieve the data taking rate expected of the experiment. For the remaining issues, fixes have been applied and spares have been put in place to deal with uncertainties in the system. Thus we conclude, the VELO has shown excellent performance and is ready for data taking. 


\section{References}

[1] The LHCb Detector at the LHC

The LHCb Collaboration

Published in Journal of Instrumentation (INST), 2008 JINST 3 S08005

[2] LHCb VELO Technical Design Report, CERN/LHCC 2001-011 (2001)

[3] Loechner, S., Schmelling, M., Beetle Refernce Manual, LHCb-2005-105

[4] L.Eklund, Control and Monitoring of VELO and Pile-Up Level 0 Electronics, EDMS document https://edms.cern.ch/document/596194.

[5] G. Haefeli et al., The LHCb DAQ interface board TELL1, Nucl. Inst. Meth. A560 (2006) 494

[6] THE CERN LARGE HADRON COLLIDER: ACCELERATOR AND EXPERIMENTS, CERN, 2008, JINST 3 S08001

[7] First Alignment of the LHCb VELO and Analysis of Beam Absorber Collision Data - Borghi, S. et al. - LHCb-2009-029 